\title{
Network Comparison of Inflammation in Colorectal Cancer and Alzheimer's Disease
}

\author{
Sungjin Park, ${ }^{1}$ Seok Jong Yu, ${ }^{2}$ Yongseong Cho, ${ }^{2}$ Curt Balch, ${ }^{3}$ \\ Jinhyuk Lee, ${ }^{4}$ Yon Hui Kim, ${ }^{1}$ and Seungyoon Nam ${ }^{1}$ \\ ${ }^{1}$ New Experimental Therapeutics Branch, National Cancer Center, Goyang-si, Gyeonggi-do 410-769, Republic of Korea \\ ${ }^{2}$ Supercomputing R\&D Center, Korea Institute of Science and Technology Information (KISTI), Daejeon 305-806, Republic of Korea \\ ${ }^{3}$ Bioscience Advising, Indianapolis, IN 46227, USA \\ ${ }^{4}$ Korean Bioinformation Center (KOBIC), Korea Research Institute of Bioscience and Biotechnology, \\ Daejeon 305-806, Republic of Korea
}

Correspondence should be addressed to Seungyoon Nam; seungyoon.nam@ncc.re.kr

Received 17 December 2014; Revised 16 February 2015; Accepted 16 February 2015

Academic Editor: Junwen Wang

Copyright (c) 2015 Sungjin Park et al. This is an open access article distributed under the Creative Commons Attribution License, which permits unrestricted use, distribution, and reproduction in any medium, provided the original work is properly cited.

\begin{abstract}
Recently, a large clinical study revealed an inverse correlation of individual risk of cancer versus Alzheimer's disease (AD). However, no explanation exists for this anticorrelation at the molecular level; however, inflammation is crucial to the pathogenesis of both diseases, necessitating a need to understand differing signaling usage during inflammatory responses distinct to both diseases. Using a subpathway analysis approach, we identified numerous well-known and previously unknown pathways enriched in datasets from both diseases. Here, we present the quantitative importance of the inflammatory response in the two disease pathologies and summarize signal transduction pathways common to both diseases that are affected by inflammation.
\end{abstract}

\section{Introduction}

Epidemiological evidence has revealed an inverse incidence between Alzheimer's disease (AD) and cancer that increases exponentially among aged cohorts $[1,2]$. However, despite the clear differences in the etiology of the two diseases, including the premature death of neurons in $\mathrm{AD}$ and evasion of apoptosis in cancer, it has been suggested that common signaling pathways are involved in the two age-associated diseases [3]. Molecular comparative surveys of the two disease states have led to speculation of roles for p53 and the Wnt signaling pathway in both cancer and $\mathrm{AD}[4]$. However, a global transcriptomic network comparison between the two diseases has yet to be completed [2].

Of interest, immune response is intimately related to both diseases [5-7]. In fact, based on an early colorectal cancer (CRC) transcriptome dataset [8], our previous study [9] found immunosuppression and immune cell infiltration even within normal-appearing cells in CRC patients. Similarly, in the brain, microglia and astrocytes involved in inflammation play a critical role in neurodegeneration $[6,7]$.
Despite continuous efforts to understand the individual molecular mechanisms of the two diseases, distinction of the global effects of immune response toward specific signal transduction usage in the two diseases has not been established. Here, we systematically inspected the two diseases representing phenotypically opposite cell fates, death and survival, by utilizing functional enrichment analysis and a systems biology approach [9]. This functional enrichment indicated that inflammatory response was significantly involved in both diseases. Subsequently, we found, by the systems biology approach, that various pathways within each disease network were comprised of common inflammationassociated genes.

\section{Materials and Methods}

2.1. Functional Enrichment Comparison of CRC and $A D$. Throughout the paper, we compared one colorectal cancer (CRC) dataset (GEO accession GSE4107) [8] with two AD datasets (GEO accessions GSE1297, GSE12685) [10, 11] from 
GSE4107 (CRC)

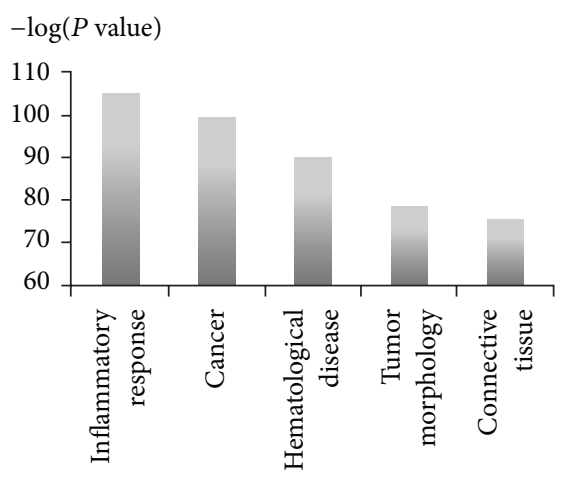

GSE1297 (AD)

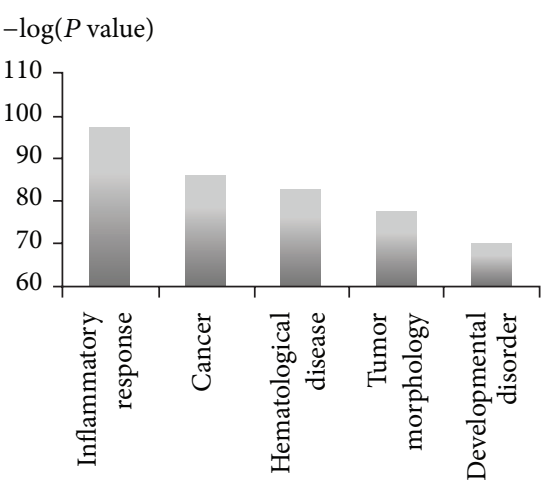

GSE12685 (AD)

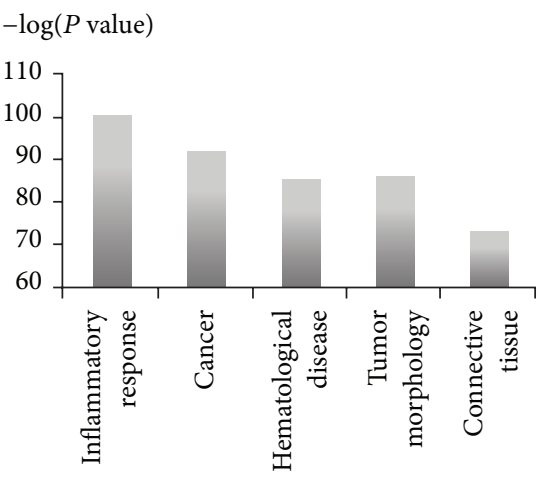

(a)
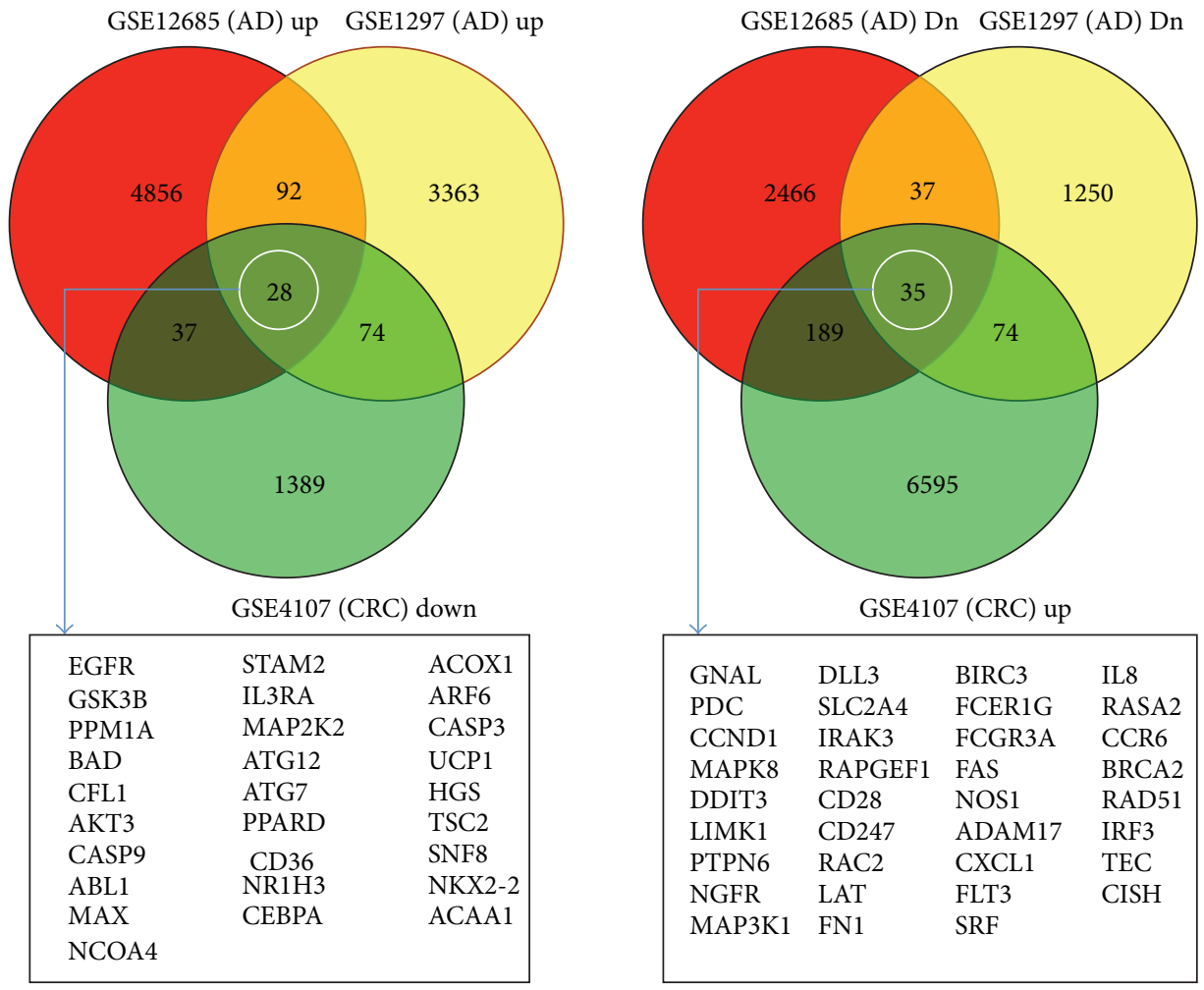

(b)

FIGURE 1: IPA functional enrichment of the CRC and the AD datasets. (a) Top 5 functional categories from "Diseases and Functions" ontology for the datasets are represented. The $y$-axis represents the minus logarithms of the $P$ values. The higher the value on the $y$-axis is, the more statistically significant it becomes. The $x$-axis represents the functional categories. (b) The common genes inversely expressed between the two diseases are indicated by white ovals (see details in Section 2). In the Venn diagrams, "GSE12685 (AD) Dn" is the downregulated gene set in AD patients versus controls. "GSE12685 (AD) Up" is the upregulated gene set in AD patients versus controls. The notation is similar to the GSE1297 (AD) dataset and the GSE4107 (CRC) dataset.

GEO (see details in Supplementary Table S1 in Supplementary Material available online at http://dx.doi.org/10.1155/2015/ 205247). We used Ingenuity Pathway Analysis (IPA, Qiagen, Valencia, CA, USA) to inspect functionally enriched terms within the IPA "Diseases and Functions" ontology, revealing the top 5 significant terms for the three datasets (Figure 1(a)). For functional enrichment analysis, we uploaded the expression fold-changes of all the genes for the three datasets into
IPA: in the CRC dataset, the expression fold-changes of patients versus controls were obtained and in $\mathrm{AD}$, the foldchanges of $\mathrm{AD}$ patients versus controls were obtained.

Since cancer and AD are phenotypically opposite (cell survival versus cell death), we obtained oppositely expressed common genes between the two diseases. Based on all the genes' fold-changes from the three datasets, we obtained the common genes as shown in Figure 1(b). 
TABLE 1: Inflammation-associated genes common to both AD and CRC show opposite expression patterns. The 16 oppositely expressed common genes (in Figure 1(b)) between $\mathrm{AD}$ and $\mathrm{CRC}$ were assigned to inflammation-associated functional terms in IPA.

\begin{tabular}{|c|c|c|}
\hline Functional category & Downregulated in $\mathrm{AD}$ and upregulated in $\mathrm{CRC}$ & Upregulated in $\mathrm{AD}$ and downregulated in $\mathrm{CRC}$ \\
\hline Chemokine & PTPN6 $^{+* \#}$, IRAK3 $^{+* \#}$, FLT3 $^{+* *}$ & $\mathrm{BAD}^{+* *}, \mathrm{CD} 6^{+* *}$ \\
\hline $\begin{array}{l}\text { Inflammation } \\
\text { relating to CRC }\end{array}$ & $\mathrm{DDIT}^{+* \#}, \mathrm{FAS}^{+* *}, \mathrm{IRF}^{+* \#}$ & \\
\hline $\begin{array}{l}\text { Inflammation } \\
\text { relating to brain }\end{array}$ & $\begin{array}{l}\mathrm{CCR}^{+* \#}, \mathrm{CD} 8^{+* *}, \mathrm{DDIT}^{+* *}, \mathrm{FAS}^{+* \#}, \mathrm{FCER}^{+* * *} \text {, } \\
\mathrm{NGFR}^{+* \#}\end{array}$ & PPARD $^{+* \#}$ \\
\hline $\begin{array}{l}\text { Cytokines relating to } \\
\text { cancer }\end{array}$ & $\mathrm{CD} 28^{+* \#}, \mathrm{FN}^{+* \#}$ & $\mathrm{ABL1}^{+* \#}, \mathrm{EGFR}^{+* *}$ \\
\hline $\begin{array}{l}\text { Cytokines relating to } \\
\text { brain }\end{array}$ & & $\mathrm{CD}^{2} 6^{+* *}$ \\
\hline
\end{tabular}

${ }^{+}$Genes detected in the CRC network from GSE4107 dataset.

* Genes detected in the AD network from GSE1297 dataset.

\# Genes detected in the AD network from GSE12685 dataset.

2.2. Network Construction of $C R C$ and $A D$. For generating networks from the three datasets, we applied our previous subpathway-based systems biology approach [9]. In brief, KEGG pathways were decomposed to all their possible paths (i.e., subpathways). In a given dataset, we applied a statistical test to each subpathway to determine whether the gene expression levels agreed with edge types (e.g., activation, inhibition) of the subpathway. Subsequently, in the dataset, we gathered the statistically significant subpathways ( $P$ values $<0.05)$ that comprised the network.

\section{Results and Discussion}

3.1. Overview. While cancer and $\mathrm{AD}$ are two of the most common diseases worldwide (15.6 million versus 7.7 million new cases per year) relating to aging, their phenotypes are opposite: cell death (neurons) in AD versus survival (mostly epithelial cells) in cancer. Also, AD patients are less susceptible to cancer and vice versa [1]. Consequently, we aimed at understanding changes at the molecular level between the two diseases. First, we inspected functional enrichment comparison of a cancer dataset (from our previous study) and the two AD datasets. Second, due to the involvement of inflammation in both pathologies $[12,13]$, we aimed to identify global network differences between the two diseases to possibly identify differential inflammation environments and differential chemokine/cytokine receptor usages. For this purpose, we selected colorectal cancer (CRC) as the cancer dataset to extend our previous result [9]. We also obtained the two independent AD datasets from GEO (Supplementary Table S1).

3.2. Functional Enrichment Comparison of CRC and $A D$ : Inflammation-Related Genes. We used Ingenuity Pathway Analysis (IPA) to perform functional pathway enrichment of early CRC and AD. IPA reported the top 5 functional categories from its "Diseases and Functions" ontology. In Figure 1(a), inflammatory response-related genes, as well as cancer-associated genes, were significantly enriched in the $\mathrm{CRC}$ and the $\mathrm{AD}$ datasets.
Figure 1(b) shows common genes that were inversely expressed between the two phenotypically opposite diseases. Considering that the biopsy tissues for the datasets contain immune cells, inflammatory response is reasonable for functional enrichment.

Out of the common genes in Figure 1(b), ARF6 was upregulated in the $\mathrm{AD}$ datasets but downregulated in the CRC dataset. ARF6, a small GTPase [14-16], regulates early endosome internalization of the protease BACE1, Beta-Site APP-Cleaving Enzyme 1. This internalization enables BACE1 to encounter and cleave intracellular amyloid precursor proteins (APPs), leading to amyloidogenic processing for the accumulation $\mathrm{A} \beta$ dimers in neurons, a hallmark of $\mathrm{AD}$ pathology [17].

CCR6 (in Figure 1(b)) was upregulated in CRC but downregulated in both $\mathrm{AD}$ datasets. CCR6 is an important surface marker of immunosuppressive immune cells in the CRC tumor microenvironment [18]. Regulatory T cells ( $\mathrm{T}_{\text {Reg }}$ cells) expressing CCR6 are recruited to a tumor mass by tumorassociated macrophages (TAMs), and tumor development is enhanced by CCR6 binding to its ligand CCL20 (CRC 1.721-fold of overcontrol in the GSE4107 dataset) secreted by tumor cells [18]. This scenario agrees with our previous result, indicating $\mathrm{T}_{\mathrm{Reg}}$ cell infiltration into normal-appearing mucosa in CRC patients [9]. Considering that $\mathrm{T}$ and $\mathrm{B}$ cells do not exist in brain, the low expression of the $T_{\text {Reg }}$ cell surface markers in $\mathrm{AD}$ patients is not surprising.

We further dissected the common genes (28 and 35 genes in white circles in the Venn diagram in Figure 1(b)) in terms of inflammation, considering that inflammatory response was the highest enrichment in all three datasets. For this purpose, we selected several terms involved in inflammation from the IPA "Diseases and Functions" ontology (see the terms and entries in Supplementary Table S2). Out of the genes common to the three datasets, 16 were oppositely expressed between the two diseases in terms of IPA inflammation-related terms (Table 1).

3.3. Network Construction of $C R C$ and $A D$. Next, we constructed molecular networks for the two diseases. By applying our previous systems biology method to the three disease 
TABLE 2: KEGG pathways associated with the 16 oppositely expressed common genes (in Table 1) in the AD and the CRC networks. From the $\mathrm{AD}$ and the $\mathrm{CRC}$ networks, pathway information of the 16 genes was obtained. The 16 genes were inversely expressed in the pathways between the $\mathrm{AD}$ and the CRC networks.

\begin{tabular}{|c|c|c|c|c|}
\hline Gene symbols & Pathways & $\begin{array}{c}\text { CRC } \\
(\mathrm{GSE} 4107)\end{array}$ & $\begin{array}{c}\mathrm{AD} \\
(\mathrm{GSE} 12685) \\
\end{array}$ & $\begin{array}{c}\mathrm{AD} \\
(\mathrm{GSE} 1297) \\
\end{array}$ \\
\hline PTPN6 & $\begin{array}{l}\text { hsa04662_B_cell_receptor_signaling_pathway; } \\
\text { hsa04630_Jak-STAT_signaling_pathway; hsa05140_Leishmaniasis }\end{array}$ & & & \\
\hline IRAK3 & hsa04722_Neurotrophin_signaling_pathway & & & \\
\hline FLT3 & hsa05221_Acute_myeloid_leukemia & & & \\
\hline DDIT3 & hsa04010_MAPK_signaling_pathway & & & \\
\hline FAS & $\begin{array}{l}\text { hsa04115_p53_signaling_pathway; } \\
\text { hsa04650_Natural_killer_cell_mediated_cytotoxicity }\end{array}$ & Up & Down & Down \\
\hline IRF3 & $\begin{array}{l}\text { hsa04622_RIG-I-like_receptor_signaling_pathway; } \\
\text { hsa04623_Cytosolic_DNA-sensing_pathway }\end{array}$ & & & \\
\hline CCR6 & $\begin{array}{l}\text { hsa04060_Cytokine-cytokine_receptor_interaction; } \\
\text { hsa04062_Chemokine_signaling_pathway }\end{array}$ & & & \\
\hline $\mathrm{CD} 28$ & $\begin{array}{l}\text { hsa04660_T_cell_receptor_signaling_pathway; } \\
\text { hsa05416_Viral_myocarditis }\end{array}$ & & & \\
\hline FCER1G & hsa04650_Natural_killer_cell_mediated_cytotoxicity & & & \\
\hline NGFR & hsa04722_Neurotrophin_signaling_pathway & & & \\
\hline FN1 & hsa04512_ECM-receptor_interaction & & & \\
\hline $\mathrm{BAD}$ & $\begin{array}{l}\text { hsa04510_Focal_adhesion; hsa05223_Non-small_cell_lung_cancer; } \\
\text { hsa05210_Colorectal_cancer }\end{array}$ & & & \\
\hline CD36 & $\begin{array}{l}\text { hsa03320_PPAR_signaling_pathway; } \\
\text { hsa04512_ECM-receptor_interaction }\end{array}$ & Down & Up & Up \\
\hline PPARD & hsa05221_Acute_myeloid_leukemia; hsa04310_Wnt_signaling_pathway & & & \\
\hline ABL1 & $\begin{array}{l}\text { hsa04012_ErbB_signaling_pathway; } \\
\text { hsa04722_Neurotrophin_signaling_pathway }\end{array}$ & & & \\
\hline EGFR & hsa05214_Glioma; hsa04012_ErbB_signaling_pathway & & & \\
\hline
\end{tabular}

datasets, we obtained CRC and AD pathogenesis networks (Supplementary Figures S1-S3). We summarized the most significant 100 subpathways for each network (Supplementary Tables S3-S5) in order to see the signaling in detail. These subpathways were assigned to various pathways in CRC and AD (Supplementary Tables S3-S5), suggesting that, in addition to inflammatory response inferred by our functional enrichment comparison, those pathways (not assigned to inflammation) remain largely unexplored in CRC or $\mathrm{AD}$. Of interest, we found pathways previously unassociated with the two diseases, including Hedgehog signaling, axon guidance, ECM-receptor interaction, and WNT signaling (Table 3). In CRC, WNT3 facilitates crosstalk between the Hedgehog and Wnt signaling pathways (Table 3). Similarly, ECM-receptor interaction was oppositely regulated between the two diseases.

3.4. Opposite Signaling Pathway Expression between CRC and $A D$ by Inflammation-Related Genes. The AD datasets were prepared from frontal cortex synaptoneurosomes and hippocampi. Both brain regions include neurons, as well as astrocytes and microglia $[19,20]$. In our previous analysis [9] of the CRC dataset, immune cells were infiltrating. Considering immune cell involvement in the two diseases and their two opposite phenotypes, different inflammationrelated molecule usage in signaling is self-evident.

So, we inspected the 16 genes' (in Table 1) differential usage of the CRC and AD networks (from Supplementary Figures S1-S3). Table 2 indicates that 16 genes were involved in extensive signaling transduction in both the CRC and AD networks, and all were inversely expressed between the two diseases.

Out of the 16 gene products, CD36 (a class B scavenger receptor) was found in microglia and vascular endothelial cells of AD patient brains [21]. Activation of CD36 and PPAR delta (gene symbol: PPARD, upregulated in both AD datasets in Table 2) resulted in FoxO1 activation in a functional study of muscle cells [22]. Considering that microglia are activated by FoxO1 [23], the two genes (CD36 and PPARD) could be involved in inflammation of $\mathrm{AD}$ patient brains.

Another intriguing observation was the opposite expression of a cell growth (antiapoptosis) gene, $M A P K$, which was upregulated in CRC and downregulated in AD, while two apoptosis pathways genes, FAS (part of the extrinsic apoptosis pathway) and $B A D$, showed the opposite pattern (up in AD and down in CRC) (Table 2). This apoptosis versus cell survival relationship has been previously postulated to explain the inverse risk correlation between malignant and neurodegenerative diseases. 
TABLE 3: Subpathways previously not associated with the two diseases. These subpathways were selected from the most significant 100 subpathways in each network. Subpathway (linear signaling flow) with fold-change (the numeral in parenthesis) of the disease group over the control group is represented in each dataset. The most significant 100 subpathways for each dataset are provided in Supplementary Tables S3-S5. The notation in the flow is "B <- A: A activates B" and "B |- A: A represses B."

\begin{tabular}{|c|c|c|c|}
\hline KEGG pathway & GSE4107 (CRC) subpathway; $P$ value & $\begin{array}{l}\text { GSE1297 (AD) subpathway; } \\
P \text { value }\end{array}$ & $\begin{array}{c}\text { GSE12685 }(\mathrm{AD}) \\
\text { subpathway; } P \text { value }\end{array}$ \\
\hline \multirow[t]{2}{*}{$\begin{array}{l}\text { Hedgehog signaling } \\
\text { (hsa04340) }\end{array}$} & $\begin{array}{l}\text { PTCH1 }(1.863)<- \text { GLI2 (2.878) |- CSNK1G1 } \\
(0.587) ; 0.000035\end{array}$ & & \multirow{2}{*}{$\begin{array}{l}\text { PTCH2 }(0.938)<- \text { GLI3 } \\
(0.682) \mid- \text { GSK3B }(1.513) ; \\
0.0015\end{array}$} \\
\hline & $\begin{array}{l}\text { WNT3 }(3.147)<- \text { GLI2 (2.878) |- CSNK1G1 } \\
(0.587) ; 0.000223\end{array}$ & & \\
\hline $\begin{array}{l}\text { Axon guidance } \\
\text { (hsa04360) }\end{array}$ & & $\begin{array}{c}\text { PAK3 }(0.732)<- \text { RAC1 } \\
(0.943) \text { |- PLXNB3 }(1.627) \\
<- \text { SEMA4C }(1.283) ; 0.0008\end{array}$ & $\begin{array}{c}\text { CFL1 }(1.157) \mid- \text { LIMK1 } \\
(0.896)<- \text { PAK4 }(0.871)<- \\
\text { RAC3 }(0.892)<- \text { PLXNA3 } \\
(0.954)<- \text { FES }(0.841) ; \\
0.0011\end{array}$ \\
\hline
\end{tabular}

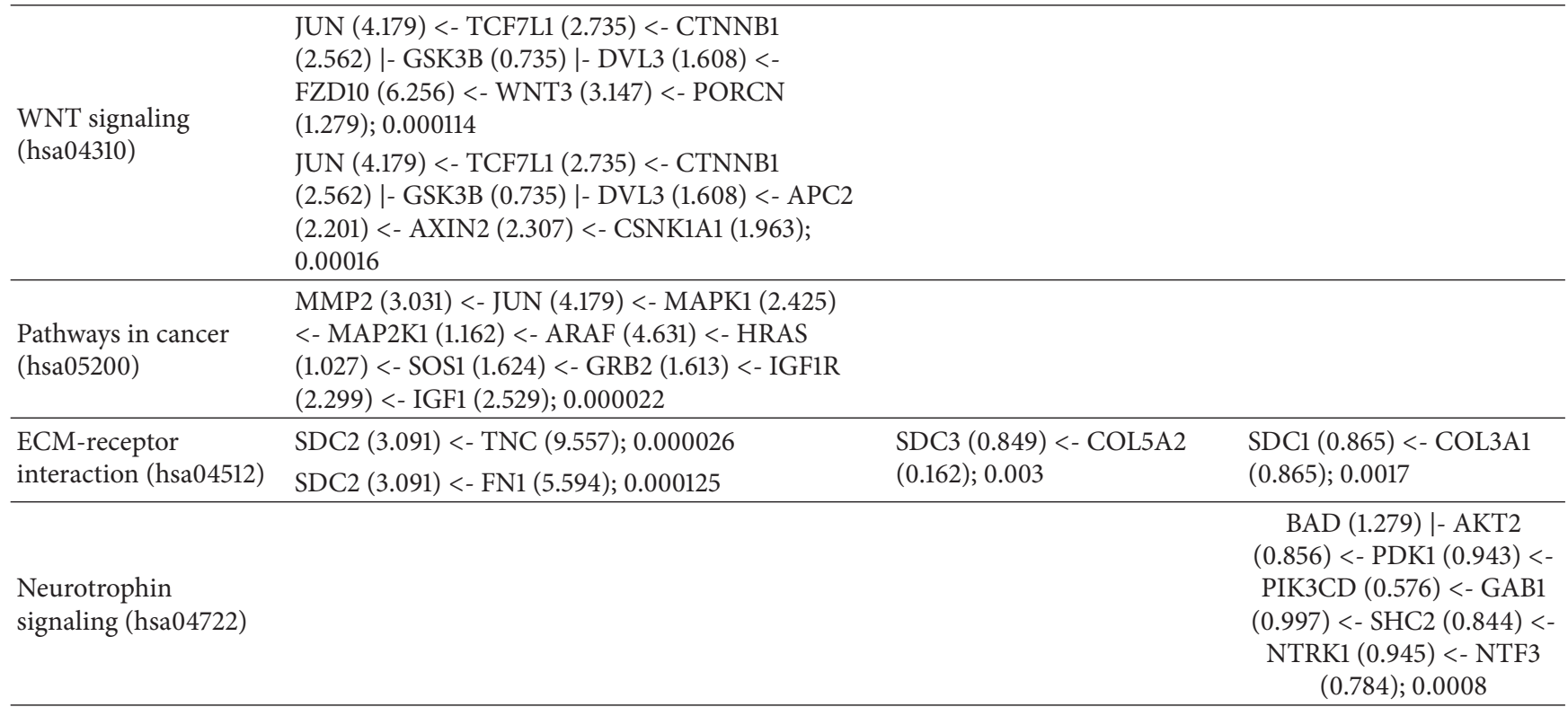

\section{Conclusions}

In general, single gene expression analysis looks into highly differentially expressed genes under a certain cutoff (e.g., $P$ value, fold-change). However, in real biological problems, signaling proteins involved in phenotype differences may not show a drastic expression-level change $[9,24]$. Also, considering that phenotype change or disease results from dysregulation of complex relationships between biological components $[25,26]$, a strict cutoff usage in single gene analysis can miss signal flow. For example, some biological entities belonging to the flow would be filtered out under a certain cutoff. Along that line, we applied our previous systems biology method [9] for describing the interdependency underlined in the diseases. In summary, we found that inflammatory response was a very important mechanism in two diseases of opposite phenotypes, that is, cancer (cell survival) and Alzheimer's disease (cell death). The inflammation-related common genes between the diseases regulated opposite gene expression in various cell signaling in the two-disease networks. In other words, the inflammation-related genes in Table 2 utilized different pathways according to the disease states, leading to different signaling transductions. Further investigation of such networks could provide knowledge into the immunological bases for the progression of both of these devastating diseases.

\section{Conflict of Interests}

Curt Balch is the Chair of Bioscience Advising, IN, USA. This does not alter the result of the study and adherence to the journal publication policy. All the authors declare no potential competing interests.

\section{Acknowledgments}

This work was supported by grants from the National Cancer Center, Republic of Korea (Grant nos. NCC-1210460 and NCC-1510141-1 to Seungyoon Nam), and Korea Institute of Science and Technology Information (KISTI) (Grant no. P14-SI-IA27 to Seok Jong Yu and Yongseong Cho), Republic of Korea. KISTI kindly provided Seungyoon Nam with a highperformance computing resource. 


\section{References}

[1] M. Musicco, F. Adorni, S. di Santo et al., "Inverse occurrence of cancer and Alzheimer disease: a population-based incidence study," Neurology, vol. 81, no. 4, pp. 322-328, 2013.

[2] K. Ibáñez, C. Boullosa, R. Tabarés-Seisdedos, A. Baudot, and A. Valencia, "Molecular evidence for the inverse comorbidity between central nervous system disorders and cancers detected by transcriptomic meta-analyses," PLoS Genetics, vol. 10, no. 2, Article ID e1004173, 2014.

[3] M. J. Devine, H. Plun-Favreau, and N. W. Wood, "Parkinson's disease and cancer: two wars, one front," Nature Reviews Cancer, vol. 11, no. 11, pp. 812-823, 2011.

[4] M. I. Behrens, C. Lendon, and C. M. Roe, "A common biological mechanism in cancer and Alzheimer's disease?" Current Alzheimer Research, vol. 6, no. 3, pp. 196-204, 2009.

[5] G. P. Dunn, C. M. Koebel, and R. D. Schreiber, "Interferons, immunity and cancer immunoediting," Nature Reviews Immunology, vol. 6, no. 11, pp. 836-848, 2006.

[6] N. J. Maragakis and J. D. Rothstein, "Mechanisms of disease: astrocytes in neurodegenerative disease," Nature Clinical Practice Neurology, vol. 2, no. 12, pp. 679-689, 2006.

[7] V. H. Perry and C. Holmes, "Microglial priming in neurodegenerative disease," Nature Reviews Neurology, vol. 10, no. 4, pp. 217-224, 2014.

[8] Y. Hong, S. H. Kok, W. E. Kong, and Y. C. Peh, "A susceptibility gene set for early onset colorectal cancer that integrates diverse signaling pathways: implication for tumorigenesis," Clinical Cancer Research, vol. 13, no. 4, pp. 1107-1114, 2007.

[9] S. Nam and T. Park, "Pathway-based evaluation in early onset colorectal cancer suggests focal adhesion and immunosuppression along with epithelial-mesenchymal transition," PLOS ONE, vol. 7, no. 4, Article ID e31685, 2012.

[10] C. Williams, R. M. Shai, Y. Wu et al., "Transcriptome analysis of synaptoneurosomes identifies neuroplasticity genes overexpressed in incipient Alzheimer's disease," PLoS ONE, vol. 4, no. 3, Article ID e4936, 2009.

[11] E. M. Blalock, J. W. Geddes, K. C. Chen, N. M. Porter, W. R. Markesbery, and P. W. Landfield, "Incipient Alzheimer's disease: microarray correlation analyses reveal major transcriptional and tumor suppressor responses," Proceedings of the National Academy of Sciences of the United States of America, vol. 101, no. 7, pp. 2173-2178, 2004.

[12] T. Wyss-Coray, "Inflammation in Alzheimer disease: driving force, bystander or beneficial response?" Nature Medicine, vol. 12, no. 9, pp. 1005-1015, 2006.

[13] A. Mantovani, P. Allavena, A. Sica, and F. Balkwill, "Cancerrelated inflammation," Nature, vol. 454, no. 7203, pp. 436-444, 2008.

[14] S. Jiang, Y. Li, X. Zhang, G. Bu, H. Xu, and Y.-W. Zhang, "Trafficking regulation of proteins in Alzheimer's disease," Molecular Neurodegeneration, vol. 9, no. 1, article 6, 2014.

[15] L. Rajendran and W. Annaert, "Membrane trafficking pathways in Alzheimer's disease," Traffic, vol. 13, no. 6, pp. 759-770, 2012.

[16] C. F. Bento, C. Puri, K. Moreau, and D. C. Rubinsztein, "The role of membrane-trafficking small GTPases in the regulation of autophagy," Journal of Cell Science, vol. 126, no. 5, pp. 1059-1069, 2013.

[17] R. Sannerud, I. Declerck, A. Peric et al., "ADP ribosylation factor 6 (ARF6) controls amyloid precursor protein (APP) processing by mediating the endosomal sorting of BACE1," Proceedings of the National Academy of Sciences of the United States of America, vol. 108, no. 34, pp. E559-E568, 2011.

[18] J. Liu, N. Zhang, Q. Li et al., "Tumor-associated macrophages recruit $\mathrm{CCR}^{+}{ }^{+}$regulatory $\mathrm{T}$ cells and promote the development of colorectal cancer via enhancing CCL20 production in mice," PLoS ONE, vol. 6, no. 4, Article ID e19495, 2011.

[19] D. Most, L. Ferguson, Y. Blednov, R. D. Mayfield, and R. A. Harris, "The synaptoneurosome transcriptome: a model for profiling the emolecular effects of alcohol," The Pharmacogenomics Journal, 2014.

[20] J. H. Choi and M.-H. Won, "Microglia in the normally aged hippocampus," Laboratory Animal Research, vol. 27, no. 3, pp. 181-187, 2011

[21] I. S. Coraci, J. Husemann, J. W. Berman et al., "CD36, a class B scavenger receptor, is expressed on microglia in Alzheimer's disease brains and can mediate production of reactive oxygen species in response to $\beta$-amyloid fibrils," The American Journal of Pathology, vol. 160, no. 1, pp. 101-112, 2002.

[22] Z. Nahlé, M. Hsieh, T. Pietka et al., "CD36-dependent regulation of muscle FoxO1 and PDK4 in the PPAR delta/beta-mediated adaptation to metabolic stress," The Journal of Biological Chemistry, vol. 283, no. 21, pp. 14317-14326, 2008.

[23] H. Dong, X. Zhang, X. Dai et al., "Lithium ameliorates lipopolysaccharide-induced microglial activation via inhibition of toll-like receptor 4 expression by activating the PI3K/Akt/FoxO1 pathway," Journal of Neuroinflammation, vol. 11, no. 1, article 140, 2014.

[24] E. Kotelnikova, N. Ivanikova, A. Kalinin, A. Yuryev, and N. Daraselia, "Atlas of signaling for interpretation of microarray experiments," PLoS ONE, vol. 5, no. 2, Article ID e9256, 2010.

[25] A.-L. Barabási and Z. N. Oltvai, "Network biology: understanding the cell's functional organization," Nature Reviews Genetics, vol. 5, no. 2, pp. 101-113, 2004.

[26] S. Nam, H. R. Chang, K.-T. Kim et al., "PATHOME: an algorithm for accurately detecting differentially expressed subpathways," Oncogene, vol. 33, pp. 4941-4951, 2014. 

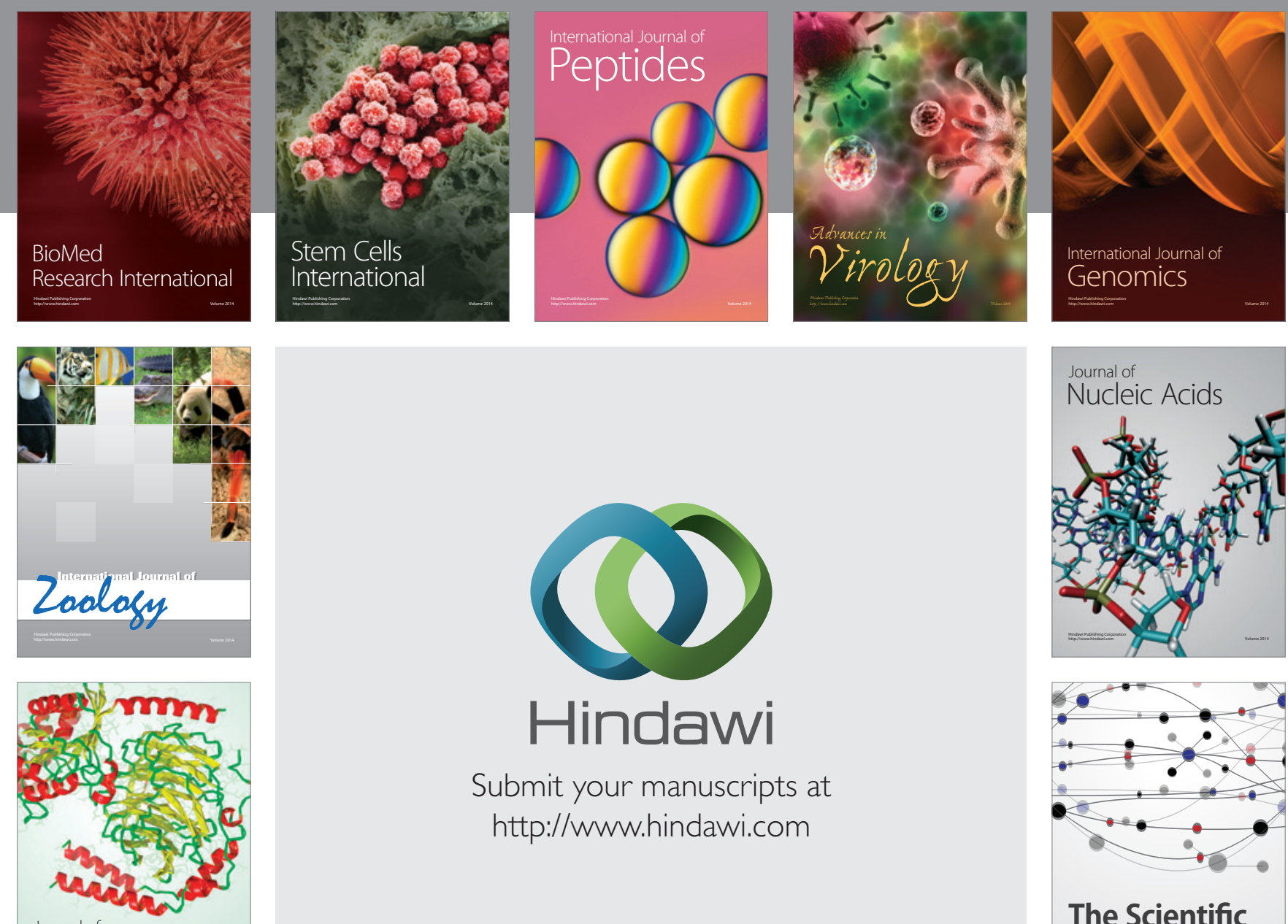

Submit your manuscripts at

http://www.hindawi.com

Journal of
Signal Transduction
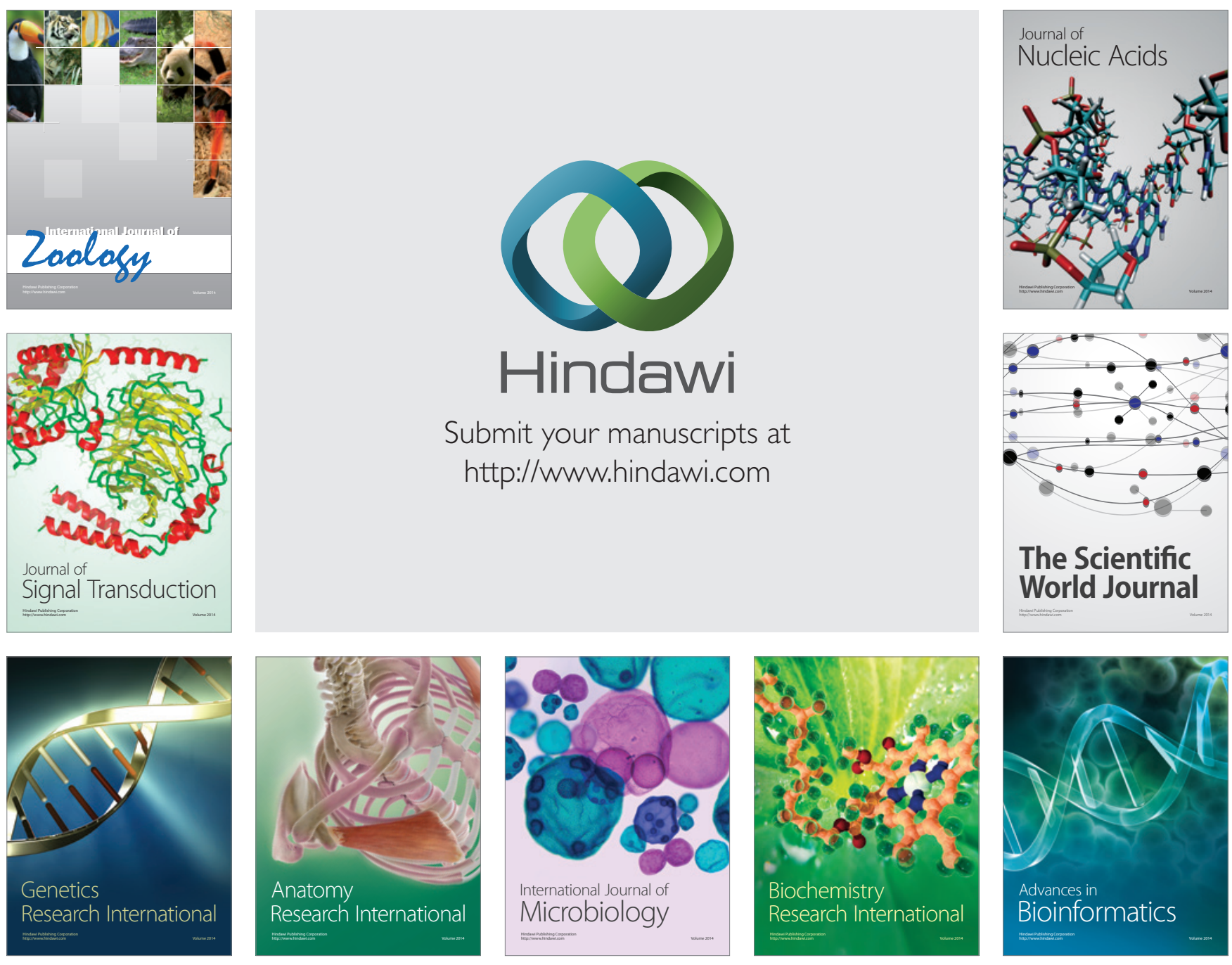

The Scientific World Journal
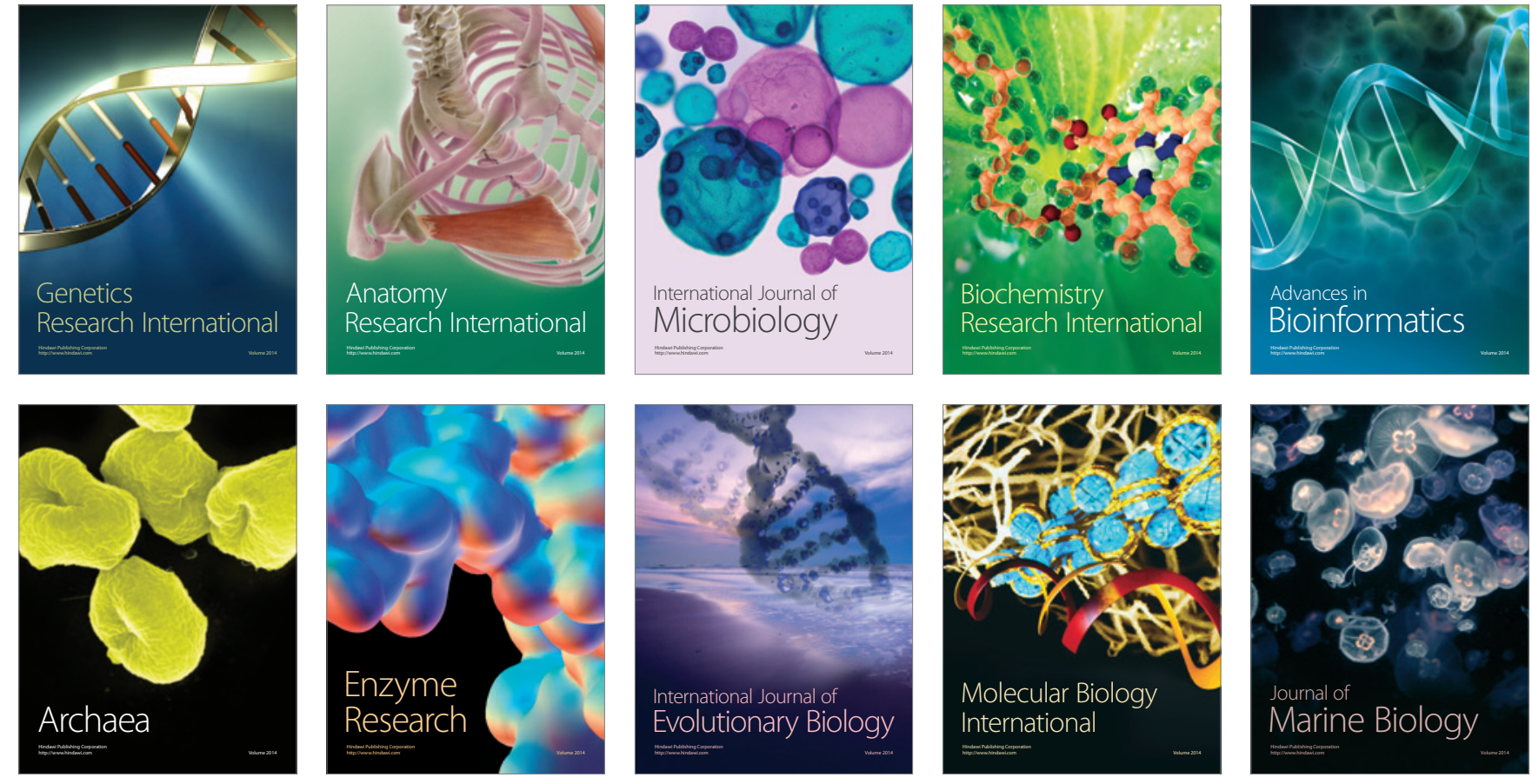